\title{
Motor learning enhances perceptual judgment: a case for action-perception transfer
}

Received: 2 June 1999 / Accepted: 20 June 2000

\begin{abstract}
Transfer from perception to action is well documented, for instance in the form of observational learning. Transfer from action to perception, on the other hand, has not been researched. Such action-perception transfer (APT) is compatible with several learning theories and has been predicted within the framework of common coding of perceptual and motor events (Prinz, 1992, 1997). Our first experiment aimed at an empirical evaluation of APT and involved motor practice of timed two-cycle arm movements on verbal command without visual feedback. In a transfer test, visual judgments of similar patterns had to be made. In addition, transfer from the visual to the motor task was studied. In Experiment 2 we separated kinesthetic aspects of motor practice from preparatory and efferent contributions to APT. The experiments provide evidence that transfer between perception and action is bi-directional. Transfer from perception to action and, more importantly, from action to perception was found. Furthermore, APT was equally pronounced for participants who had actively practiced movements during training and for passive participants who had received merely kinesthetic feedback about the movement. This kinesthetic-visual transfer is likely to be achieved via visuomotor-kinesthetic matching or via timekeeping mechanisms that are involved in both motor and visual performance.
\end{abstract}

\footnotetext{
H. Hecht ( $\square)$

Massachusetts Institute of Technology, MIT Man-Vehicle Lab, 77 Mass Ave., Bldg. 37-219, Cambridge, MA 02139, USA e-mail: hecht@mit.edu

S. Vogt $(\bowtie)$

Department of Psychology, Lancaster University,

Lancaster LA1 4YF, UK

e-mail: S.Vogt@lancaster.ac.uk

W. Prinz

Max Planck Institute for Psychological Research, Amalienstrasse 33, D 80799 München, Germany e-mail: prinz@mpipf-muenchen.mpg.de
}

\section{Introduction}

We can educate or shape our competencies for performing actions by means of watching other people's performance. This observational learning, as well as instances of imitation that operate on shorter time scales such as immediate or deferred imitation, requires some form of communication between perceptual and motor processes. A number of theoretical approaches assume that the perception of an action activates internal cognitive mechanisms that can also be used in the generation of action (Bandura, 1986; Meltzoff \& Moore, 1997; Meltzoff \& Prinz, in press). We refer to this phenomenon as perception-action transfer (PAT) as a general term that includes 'observational learning' as used in work with human participants, and 'imitation' (of novel actions) as used in work with non-human primates (e.g., Byrne \& Russon, 1998). Here we investigate whether the reverse phenomenon also holds true. That is, can our action competencies educate, or shape our perceptual skills?

Such transfer from action to perception might reflect common structural properties of visual and motor memory, and it can elucidate the process of motor learning. From a theoretical perspective, action-perception transfer (APT) is compatible with all learning models that allow for amodal or intermodal learning. APT has been explicitly predicted by the theoretical framework of common coding (Prinz, 1992, 1997). According to this principle, the final stages of perception and the initial stages of action control share a domain of coding where planned actions are represented in the same format as perceived events. One of the implications of this approach is that, under appropriate conditions, perceived environmental events can induce certain actions by way of similarity or feature overlap. If perception and action share the same codes, it must also be expected that changes in these codes that are due to motor learning are reflected in corresponding changes in perceptual skills. However, since an empirical 
demonstration of APT is compatible with a number of learning models (except pure intramodal learning), we do not intend a critical test of the theory of common coding in this study. Our main objective is to explore whether APT can be demonstrated at all.

In the following, we briefly delineate the research deficit regarding APT within the wider area of visuomotor interactions. In Table 1 these interactions are classified along two dimensions. The first dimension, time scale, differentiates between concurrent, or shortterm interactions and long-term transfer phenomena. The other dimension, direction of transfer, indicates whether perceptual processing involves motor processing (visuomotor coupling) or vice versa (motor-visual coupling). This taxonomy is meant to be descriptive and does not override the existing local theories. On the short time scale, evidence for visuomotor coupling during action observation, and evidence for concurrent effects of motor action on perception have been found in a number of studies. On the long time scale, observational learning is a well-established phenomenon, while APT has not yet been documented.

In the next two sections, we briefly review the existing evidence for visuomotor interactions. Concurrent motor-visual effects can be seen as equivalents of APT on a shorter time scale. However, also the evidence for the involvement of motor processes in action observation provides an important background for our prediction of APT. If motor processes are indeed involved in perceptual tasks, we may reach the somewhat counterintuitive conclusion that engaging in a motor action directly improves the related perceptual skill.

\section{From perception to action}

PAT is well-studied on the long time scale of observational learning and needs only be briefly mentioned here (for reviews see McCullagh, Weiss, \& Ross, 1989; Vogt, in press-b). Its classical models (e.g., Bandura, 1986; Carroll \& Bandura, 1990) assume distinct stages of perceptual processing and subsequent visuomotor matching. More recently, Vogt (1995) found that the temporal consistency in producing sequential arm movements improved to the same extent through men-

Table 1 Effects of perception on action have been established on all time scales. Motor-perceptual effects, on the other hand, have only been studied on a short time scale. Do longer-term effects of action on perception also occur? Such learning effects are referred to as action-perception transfer (APT)

\begin{tabular}{lll}
\hline & Short time scale & Long time scale \\
\hline Perception $\rightarrow$ action & $\begin{array}{l}\text { Visuomotor coupling } \\
\text { (e.g., imitation)/ } \\
\text { interference }\end{array}$ & $\begin{array}{l}\text { Observational } \\
\text { learning }\end{array}$ \\
Action $\rightarrow$ perception & $\begin{array}{l}\text { Motor-visual } \\
\text { coupling/interference }\end{array}$ & $\begin{array}{l}\text { Action- } \\
\text { perception } \\
\text { transfer ? }\end{array}$ \\
\hline
\end{tabular}

tal, observational, and physical practice. Whereas the equivalence of motor imagery and physical practice could be expected from earlier behavioral and neurophysiological work (see Jeannerod, 1994, 1997; Jeannerod \& Decety, 1995), the equivalence of observational and physical practice was unexpected. It indicates that motor structures are already involved during movement observation and thus provides a theoretical alternative to distinct stage models.

Also on the shorter time scale of briefly deferred and immediate imitation, motor processes are involved in action observation. For instance, when participants had to reproduce a cyclical movement whose relative timing varied from trial to trial, reproductions performed directly after viewing the display were at least as accurate as imitation after various intermediate forms of rehearsal (Vogt, 1996). Thus, information for action was available directly after model observation and did not require further elaboration. More recently, Brass, Bekkering, and Prinz (in press; see also Bekkering, in press; Prinz, in press) showed that displays of finger and mouth movements can interfere with motor preparation of similar movements even if the display is irrelevant to the task. These experiments demonstrate that action displays access motor preparatory processes in a fast and automatic manner, as shown before for spatial stimulus attributes in stimulus-response compatibility (e.g., Hommel \& Prinz, 1997). Other action-related object properties such as orientation and size have also been shown to automatically prime corresponding actions (Craighero, Fadiga, Umiltà, \& Rizzolatti, 1996; Tucker \& Ellis, 1998).

A direct comparison of visuomotor latencies in imitative and object-guided performance has been undertaken by Vogt (in press-a, and manuscript in preperation). Using a reaching task, it was found that responses to shifts of a target's location were equally fast as responses to shifts in the direction of an observed human hand while reaching for a target. Both latencies were significantly shorter than equally informative color cues. Since responses to shifts of object location are amongst the fastest visuomotor couplings in humans and are known to be processed in the dorsal cortical pathway (Arbib, 1997; Jeannerod, 1997; Jeannerod, Arbib, Rizzolatti, \& Sakata, 1995), the similarly short latencies for model-guided responses indicate that the same pathway may be used in this simple form of imitative guidance of reach direction.

These and further behavioral findings (Shiffrar \& Freyd, 1993; Viviani \& Stucchi, 1992a, 1992b) are complemented by a number of neurophysiological studies that demonstrate the involvement of motor cortical structures in action perception. The initial finding of 'mirror neurons' in the monkey premotor cortex that fire both during action production and action observation (DiPellegrino, Fadiga, Fogassi, Gallese, \& Rizzolatti, 1992) has been followed by a number of brain imaging studies that support the idea of a mirror system in humans (for reviews see Gallese \& 
Goldman, 1998; Grezes, Costes, \& Decety, 1998; Meltzoff \& Prinz, in press; Rizzolatti, Fadiga, Gallese, \& Fogassi, 1996; Vogt, in press-b). Thus, both behavioral and neural evidence for visuomotor couplings during action observation is available.

\section{From action to perception}

When motor processes contribute to perception, we should not only expect longer-term consequences in the sense of the hypothesized transfer effects (APT), but also concurrent motor-perceptual interactions. Thus far, only concurrent effects have been demonstrated. For instance, Ishimura and Shimojo (1994) described a series of experiments where perceived motion was biased by concurrent hand movements. When observers viewed bistable apparent motion displays that could yield impressions of vertical or horizontal motion, perceived motion directionality was highly correlated with the direction of movements of their hands that were occluded from view. The authors interpreted this "action capture" as possibly subserving visually guided hand movements by a selective enhancement of visual sensitivity to the plane and direction of motion of the intended hand movement. In a more recent study, Wohlschläger (2000) found similar results when using rotational rather than the unidirectional motor and perceptual tasks employed by Ishimura and Shimojo. Interestingly, Wohlschläger also showed that planned hand movements, that were only executed after the visual judgment, were sufficient to bias apparent motion perception. Ishimura and Shimojo, on the other hand, found no facilitating effects of imagined hand movements.

In line with the results of Wohlschläger (2000), Craighero, Fadiga, Rizzolatti and Umiltà (1999) showed that the mere preparation of reaching to grasp a bar in a certain orientation facilitated responses to a congruent visual stimulus. This facilitation was not only observed for reach onset latencies (Craighero et al., 1996) but also when the response was a neutral footswitch press. While the former finding could be due to both visuomotor and motor-visual priming, the authors interpreted the latter as a genuine motor-visual priming effect where motor planning directs the visual-attentional processing of movement-relevant stimuli.

When motor preparation and perceptual identification serve two independent tasks, feature overlap may lead to interference rather than facilitation effects. A corresponding scenario was studied by Müsseler and Hommel (1997). They found that the identification probability of an arrow was impaired when participants were simultaneously preparing a keypress that was compatible with that arrow but irrelevant for the identification task. Since both tasks had visual and motor components, they demonstrated in a follow-up experiment that this inverted compatibility effect was neither due to intra-perceptual interference nor to intra-motor preparatory interference, but that it was a genuine mo- tor-visual effect. A detailed discussion of concurrent motor-visual interactions can be found in Müsseler (1999).

In sum, there is an asymmetry in the study of PAT and APT. Perception-action transfer on the long time scale (observational learning) as well as on shorter time scales (visuomotor couplings) is well documented by imitation tasks and by interference tasks that involved action displays. In contrast, motor-visual effects have only been shown with concurrent priming and interference paradigms. In the present study, we explore the existing gap of longer-term motor-perceptual interactions or APT. We evaluate whether effects of motor learning manifest themselves in a perceptual equivalent of the motor task. It should be noted that the dichotomy of visuomotor and motor-visual effects does not necessarily imply two distinct functions, since the role of at least some of the reported visuomotor couplings may well be to constrain and thus enhance perception, which is just how Ishimura and Shimojo interpreted their motor-perceptual effects.

The two basic tasks used in our experiments were a motor production task and a visual judgment task. For the former, we have chosen a similar task as used by Vogt (1996), namely the production of two consecutive sine-wave arm movements. Participants performed different relative timings of the two movements as indicated by verbal command in each trial under exclusion of visual feedback. We matched this task with a visual task that required judging the relative timing of such movements displayed on a monitor. Practice in both tasks involved verbal feedback about the accuracy of production or judgment. Both transfer from action to perception (APT) and from perception to action (PAT) were assessed. The empirical demonstration of PAT in Experiment 1 confirmed that our visual task did indeed involve a motor component. More importantly, Experiment 1 also provided clear evidence for APT in the sense of enhanced perceptual discrimination following motor practice without vision. In Experiment 2, we tried to narrow down the nature of APT. It could be that APT is related to the planning and execution components of motor performance. Alternatively, APT could derive from an enhanced ability to interpret the kinesthetic signals that arise during motor practice. We found that the latter alternative provides a sufficient account for APT in our task.

\section{Experiment 1}

The first experiment was designed to study the occurrence of APT in a simple laboratory task. A task had to be found that was structured similarly in its visual and its motor format. Also, to test APT in a meaningful way, the participants have to acquire a motor skill without being visually exposed to the task. To achieve this, the task had to be sufficiently simple to be based exclusively on verbal instruction and feedback. Finally, the task 
should allow learning progress within both its visual and motor analogs. We chose timed sequences of sinusoidal arm movements among possible candidates fulfilling all three requirements. The motor task consisted of producing timed movements using a mechanical lever, and the visual task required judgments of time ratios of sinusoidal bar movements displayed on a monitor.

\section{Method}

Participants. Forty-eight right-handed college-age students (24 male, and 24 female) at Ludwig-Maximilians-Universität, Munich, were paid for their participation. None of them had prior experience with the lever task.

Design. A between-subjects learning paradigm was used as shown in Table 2. Each of three groups received different practice tasks, while everyone was subsequently subjected to the same visual and motor tests. The first group received only motor practice, the second group received visual practice, and the control group received motor practice in a (non-dynamic) distance judgment task. The between-groups practice manipulation together with identical visual and motor tests was chosen to assess the differential effects of motor and visual practice on performance both within and between modalities. Comparing the visual test results between groups should reveal potential APT, whereas comparing motor test results should reveal PAT effects. Participants were randomly assigned to the three practice conditions with the constraint that an equal number of male and female participants were in each group.

Motor task and apparatus. The motor task was to produce two cyclical arm movements with a certain relative timing specified by verbal command. A mechanical lever (wood, $48 \mathrm{~cm}$ long) was mounted to a table with an aluminum bearing such that it was free to move in the horizontal plane. The participant's elbow was placed directly above the pivot point, while an adjustable pin at the tip of the lever was locked into position between the index and middle fingers of the participant's right hand. Seat height was adjusted to allow participants to rest their arm on the lever comfortably. A Hewlett Packard 386/25 personal computer (PC) with a Data Translation $2821 \mathrm{~A} / \mathrm{D}$ board was used to collect angular position data (spatial resolution $2^{12}$ points, effective sampling rate $200 \mathrm{~Hz}$ ). These data were obtained from a precision potentiometer attached to the pivot of the lever.

Each movement cycle started at a lever position of $30^{\circ}$ (with $0^{\circ}$ being in the fronto-parallel plane), then moving to the right to about $90^{\circ}$ (the lever pointing straight ahead from the participant) and back to the start position. One trial consisted of two cycles back-to-back. The task was to produce a smooth movement with the prescribed amplitude and taking $800 \mathrm{~ms}$ for the first cycle and a given proportion (between $50 \%$ and $200 \%$ ) for the second cycle. A second PC was used to compute time ratios for verbal feedback during motor practice. From the recorded position-time curve, its first two derivatives were estimated (using a Kaiser filter with a cutoff frequency of $10 \mathrm{~Hz}$ and a transition width of $8 \mathrm{~Hz}$ ) and were shown to the experimenter for control purposes. Intervals between peak acceleration and peak deceleration were then calculated for both cycles, and verbal feedback was given to the participant as a single $\%$ value based on the ratio between the second and the first interval.
Visual task and apparatus. The visual task was to judge the temporal ratio of two-cycle sinusoidal motions of a vertical bar. Participants were seated behind a desk and in front of a Lucius and Baer MST 6051 analog display $(40 \times 30 \mathrm{~cm})$, which was controlled by the same PC and data translation board as used in motor practice. A display rate of $800 \mathrm{~Hz}$ was chosen to approximate continuous motion. A bright vertical white bar (4 cm long) moved back and forth on each movement cycle, starting from a point $12 \mathrm{~cm}$ to the left of the screen center to a point $12 \mathrm{~cm}$ to the right of the center and then back to the start position. Within each cycle the motion accelerated towards the center of the screen and then decelerated toward the turning points in a sinusoidal fashion. Each trial consisted of two cycles identical in amplitude $(24 \mathrm{~cm})$ with no interval between cycles. The first cycle always took $800 \mathrm{~ms}$, while the second cycle varied between 400 and $1600 \mathrm{~ms}$, thus producing time ratios (cycle 2/cycle 1) between $200 \%$ (slowest second cycle) and $50 \%$ (fastest second cycle). The viewing distance was $125 \mathrm{~cm}$, leading to an amplitude of approximately $11^{\circ}$ of visual angle. The monitor was in an upright position centered at the observer's line of sight. In the visual practice trials, feedback about the presented time ratio was given after each display.

\section{Practice and test procedures}

Motor practice. The table with the mechanical lever device was shown and the two-cycle movement explained in general terms. Then, the participants was seated and blindfolded. The right arm was fixed to the lever with two Velcro straps going around the forearm close to wrist and elbow respectively. The experimenter then moved the lever to the start position and guided a standard movement consisting of two smooth cycles of equal duration $(800 \mathrm{~ms})$. Participants were given between 6 and 12 familiarization trials with timing and amplitude feedback until they could reliably reproduce this movement.

Next, the time-ratio concept was explained by means of a drawing representing the constant first cycle and the variable second cycle. Throughout the experiment it was verified that participants correctly understood the translation of percent values into time ratios. A $150 \%$ movement was practiced three times. It was emphasized that producing a correct 'ratio' between first and second cycle had priority over keeping cycle 1 constant in absolute terms. The practice trials were presented in random order varying in steps of $5 \%$ from $50 \%$ to $100 \%$ and in steps of $10 \%$ from $100 \%$ to $200 \%$. Participants were also asked to join the two cycles into one smooth movement at all times.

Three practice blocks of 21 trials each were performed. The experimenter verbally communicated the required \% value and participants started at their convenience with the movement. Thus, they could mentally prepare for the required time ratio. They were encouraged to repeat a trial whenever they felt they had markedly deviated from the intended movement. Trials were also repeated when the allotted time of $3 \mathrm{~s}$ per movement was exceeded. Less than $15 \%$ of all trials had to be repeated during the first practice block and less than $5 \%$ in the following practice blocks.

To ensure a precise start position of the lever, a high-pitched tone sounded while the lever was in any location but the start position. Thus, the start position had to be found by moving the lever back and forth until it came to rest in the no-sound zone, which covered about $0.5^{\circ}$. After each practice trial, verbal feedback about the actually produced time ratio was given as explained above. In about $2 \%$ of all trials artifactual zero crossings in the second derivative had to be excluded by the experimenter to
Table 2 Design used in Experiment 1. Each of 16 participants per group was subjected to three phases. Only the practice phase (1) differed between participants

\begin{tabular}{llll}
\hline & Motor group & Visual group & Control group \\
\hline Phase 1 (63 trials) & Motor practice & Visual practice & Control practice \\
Phase 2 (42 trials) & Visual test (APT) & Visual test & Visual test \\
Phase 3 (42 trials) & Motor test & Motor test (PAT) & Motor test \\
\hline
\end{tabular}


achieve adequate estimates. Feedback about absolute time and amplitude was only given when any of these exceeded a tolerance range of $\pm 30 \%$.

Visual practice. Participants in this condition were told that they first had to practice judging visual movements and would then be tested on related tasks. First, the standard motion of two identical cycles $(100 \%)$ was explained and presented six times. Next, the same representation of duration ratios as used in the motor practice group was shown and explained. Participants were told that the first cycle would always be the same, while the second varied between $200 \%$ and $50 \%$ of the first cycle's duration. The same time ratios were used as for the motor group.

Next, participants were shown a $150 \%$ motion twice. Then they were asked to judge three blocks of 21 randomly ordered trials. On each trial, the complete two-cycle motion was presented twice, with a short (1 s) time interval between presentations. Feedback about the time ratio was delayed for about $7 \mathrm{~s}$ to equate the time between movement and feedback for all practice conditions.

Control practice. The control task was selected to provide similar experience with ratio judgments, while preventing exposure to timing. Participants in this group performed a haptic control task. A 50-cm-long linear slide ruler was used to learn how to produce distance ratios. After briefly looking at the slide ruler, participants were blindfolded. A sliding marker could only move leftward from its start position. At $15 \mathrm{~cm}$ to the left, a fixed haptic pointer indicated a distance labeled as $100 \%$. Participants had about seven trials to familiarize themselves with reproducing that distance. On each practice trial, they could first feel the pointer with their left hand. Then, with the left hand no longer touching the apparatus, they used their right hand to move the sliding marker to an equivalent distance $(100 \%)$, back to the starting position, and after a brief pause to the designated distance between $50 \%$ and $200 \%$ of the standard. For three blocks of 21 trials, the experimenter announced the required percentage of the standard distance. After each trial, verbal feedback about the actually produced distance was given with a delay comparable to motor and visual practice.

Visual test. All participants received the same visual test. As in visual practice, they were shown the standard two-cycle vector display with a time ratio of one $(100 \%)$ first. The same procedure as in visual practice was used to explain how to judge motion ratios in terms of \% values. Participants from the control practice group were instructed more carefully, since this was the first time they encountered time ratios. All participants made duration ratio judgments for two blocks of 21 trials each. The trials were identical to the ones used in visual practice with regard to all stimulus parameters but were presented in different random orders. On each trial, the complete two-cycle motion was presented twice before a $\%$ value had to be written on a prepared sheet of paper. Feedback was not provided. A short break was taken between the two blocks.

Motor test. Following the visual test, all participants underwent a motor test. Identical to the procedure in motor practice, they were first familiarized with the standard two-cycle movement for about seven trials. Participants belonging to the motor practice group usually needed only two to three trials to remind them of the procedure. Then, the duration ratio concept was re-explained. All participants were blindfolded while they produced lever movements with instructed duration ratios ranging between $50 \%$ and $200 \%$. Two motor test blocks of 21 trials were carried out without verbal feedback about the time ratio. Participants were alerted when their movements deviated from the desired amplitude by more than $30 \%$. Trials in which participants exceeded the maximal recording time of $3 \mathrm{~s}$ were repeated. This happened in less than $10 \%$ of trials.
Results

Taken together, visual and motor tests revealed positive transfer effects from perception to action as well as from action to perception. Overall error and variability were significantly smaller for the experimental groups compared to the control group. In the visual test, participants benefited from prior motor practice, and in the motor test, participants benefited from prior visual practice. Since the scores in the visual and motor tasks are fundamentally different they cannot be compared in a straightforward manner. Thus, the comparison to the control group is particularly important, and we hypothesize its results to differ from both experimental groups. Consequently, we present separate statistical analyses for visual and motor practice as well as for visual and motor tests.

\section{Practice}

To measure performance during motor and visual practice, data were analyzed according to the same procedure as used for verbal feedback. That is, from the motor performances, intervals between peak acceleration and peak deceleration of each cycle were determined and their ratio (cycle 2/cycle 1) was calculated. The empirical (motor or visual) duration ratios were then compared to the required or displayed ratios using the root mean square error (E) as an overall error measure, along with its constituent constant and variable error components (Schmidt 1988), $\mathrm{E}^{2}=\mathrm{CE}^{2}+$ $\mathrm{VE}^{2}$.

The constant error (CE) is the average signed deviation between target duration ratio and produced/judged duration ratio, whereas the variable error (VE) reflects the variability in participant responses across trials in a block, that is, it measures the variability around CE. Although a further decomposition of VE into slope and residual error is possible (Vogt 1996), the three error measures mentioned above are fully sufficient for the present purposes.

Strong improvements within the three visual practice blocks were found in a one-factorial repeated measures ANOVA with $\mathrm{E}$ as dependent measure, $F(2,30)=$ $14.03, p=0.0001$. The visual group improved consistently over the three blocks as indicated by a drop of total error E from 20.26 in Block 1 to 13.15 in Block 3, $F(1,15)=17.53, p=0.0008$. Variable and constant error also decreased significantly with practice, $F(2,30)=14.17, p=0.0001$ for $\mathrm{VE}$, and $F(2,30)=$ $3.40, p=0.0467$ for CE.

The motor practice group improved significantly in variable error and only marginally in total error. That is, a repeated measures ANOVA was not significant for total error, $F(2,30)=2.16, p=0.13$, and the drop in error between the first (28.63) and the third motor practice block (23.95) was only marginally significant, $F(1,15)=3.68, p=0.0744$. However, practice had a 
significant effect on VE, $F(2,30)=3.70, p=0.0365$. VE reduced from 27.77 during the first practice block to 22.02 in Block 3, $F(1,15)=8.68, p=0.01$. CE was not significant.

In sum, both experimental groups showed significant improvements during practice in their respective modalities. For motor practice, but also for visual practice, the effect was mainly carried by a reduction in variability.

\section{Visual test}

Figure 1 depicts the total errors (E) made by the three groups in the visual test. The error data were computed according to the same procedure as for the practice blocks. Repeated measures ANOVAs were run separately for the different error measures. The overall error $\mathrm{E}$ in the visual test varied significantly between the three groups, $F(2,45)=13.69, p<0.001$. The following contrasts between groups were obtained within the same analysis. As expected, the visual practice group had smaller $E$ values than the control group, $F(1,45)=26.23, p<0.001$. The motor practice group also performed considerably better on the visual task than the control group, $F(1,45)=12.19, p<0.001$. Motor and visual practice groups did not differ significantly, with motor practice producing only marginally larger errors than visual practice, $F(1,45)=2.66$, $p=0.11$. Thus, the positive transfer effect of motor practice approached the effects of intramodality learning in the visual test.

$\mathrm{CE}$ and $\mathrm{VE}$ were analyzed in the same manner as E. Transfer effects for the motor practice group were comparable for all error measures. While overall group differences were large for $\mathrm{CE}, \quad F(2,45)=10.33$, $p<0.001$, there was virtually no difference in CE between visual $(-6.17)$ and motor $(-7.43)$ practice groups, $F(1,45)=0.71, p=0.41$, whereas control group errors

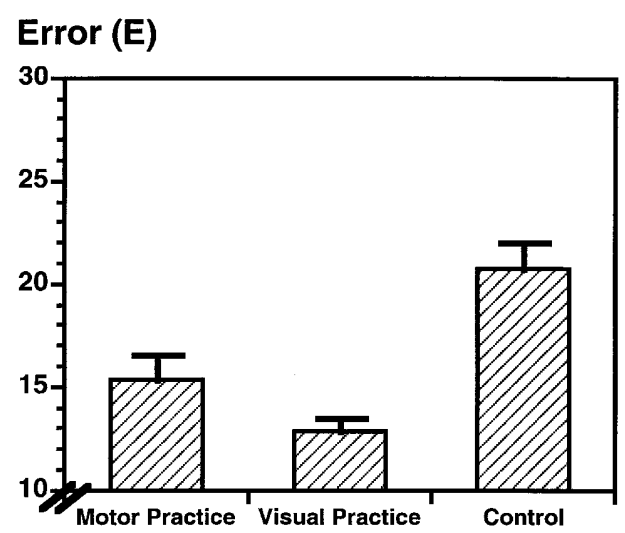

Fig. 1 Total Error $(E)$ in the visual test of Experiment 1. Participants who practiced a motor task while deprived of visual information showed transfer to a subsequent visual task (actionperception transfer). They performed almost as well as the visual practice group were large (-12.59). All participants underestimated the true ratio, that is, they judged the ratio between second and first cycle to be smaller than it actually was. This bias to underestimate the duration of the second cycle was equally attenuated in the visual and motor practice groups. The picture was similar for VE. A large main effect of group was found, $F(2,45)=9.17, p<0.001$. The visual practice group (10.9) produced a somewhat smaller VE than the motor practice group (13.0), but this difference did not reach significance, $F(1,45)=2.95, p=0.09$. Further, no significant differences were found between the first and the second block of visual test trials.

Figure 2 compares the performance of the motor practice group to the control group in the first visual test block. The scatter plot depicts judged ratios as a function of displayed ratios. Scores were more accurate and less variable for the motor practice group.

\section{Motor test}

Visual practice led to improved motor test performance as depicted in Fig. 3. A repeated measures ANOVA on E was significant, $F(2,45)=6.72, p=0.003$. Contrasts within the same analysis showed that the visual practice group was significantly better than the control group, $F(1,45)=4.28, p=0.044$, and only marginally worse than the motor practice group, $F(1,45)=2.52$ $p=0.119$. The contrast between motor and control group was highly significant, $F(1,45)=13.36$, $p<0.001$. CE measures were analogous to the visual test results, $F(2,45)=5.50, p=0.007$. The motor and visual practice groups did not differ significantly from each other (-1.10 and 3.94), but both were considerably

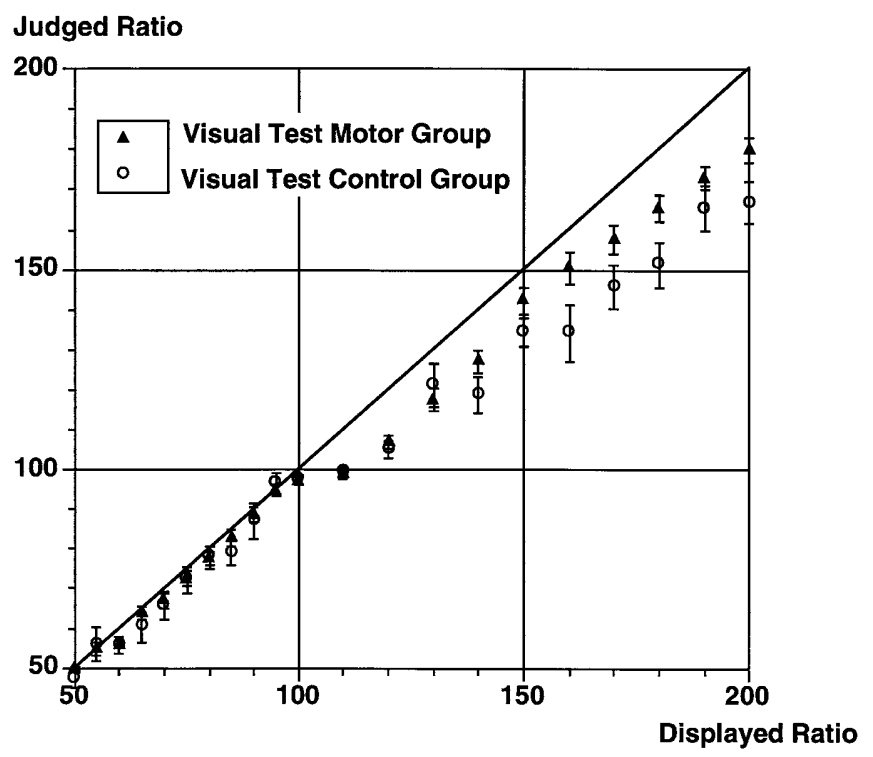

Fig. 2 Relationship between displayed and rated \% values in the visual test of Experiment 1. The control group has less accurate and more variable judgments than the motor practice group 


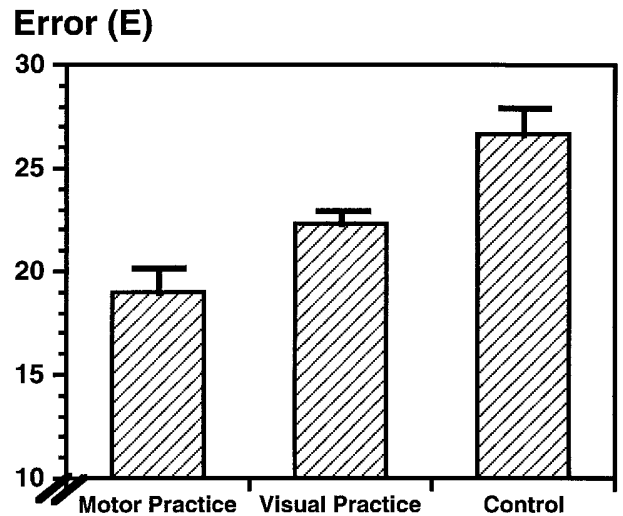

Fig. 3 Total Error $(E)$ in the motor test of Experiment 1. Participants who practiced a purely visual task benefited from this practice when they had to carry out movements that were similar in structure (perception-action transfer). They produced lower overall errors than the control group

better than the control group (9.65), $F(1,45)=10.98$ $p=0.002$. A significant main effect was also found on the variable error measure, $F(2,45)=7.03, p=0.002$. However, the visual practice group produced a larger VE (21.39) than the motor practice group (16.52), $F(1,45)=7.43, p=0.01$. On all error measures, there were no significant differences between the first and second motor test block.

\section{Discussion}

We have found evidence for PAT and, more importantly, for APT. The demonstration of APT is, to our knowledge, unique, whereas PAT has been shown in previous experiments on observational learning. The evidence for PAT from our visual task to our motor task is also relevant for interpreting APT, as will become clear in the following.

The results support at least two - not mutually exclusive - mechanisms for our main finding of an enhanced perceptual discrimination following motor practice, or APT. First, the concept of common representations that are shared between different processes is supported. The key assumption here is that a representational structure on a higher level of coding can be accessed from action and from perception and thus enables the observed transfer. Second, concurrent interactions between perceptual and motor processes, not implied by the first approach, explain the same results in a somewhat different manner. According to this interpretation, APT is due to the contribution of the same motor preparatory processes that were involved in motor practice. In other words, APT as observed in the visual task would reflect the operation of visuomotor couplings with motor components that were enhanced in the previous motor practice. Fortunately, the implication that motor processes are involved in our visual task is supported by the finding that visual practice transferred to motor production (PAT). Without assuming some form of motor involvement during visual practice, one should expect poorer performance in the initial motor test trials and, accordingly, improvements between both parts of our motor test due to gradual correction processes. Such improvements were not found. Finally, the fact that evidence for visuomotor couplings was found in tasks that were similar to the present one (Vogt, 1995, 1996, in press-a) supports our interpretation of APT as arising from such couplings during the visual test.

While our findings provide clear evidence for APT, they also indicate some limits of this kind of transfer. The positive transfer found on constant and variable error measures was not always complete. That is, intramodality (visual-visual) learning was more efficient than motor-visual transfer. Although this may, at first sight, be seen to contradict our proposal that motor practice is a direct means of improving perceptual skills that involve motor processes, it should be clear that visual learning is not confined to this motor component, and even if motor encoding played a crucial role, it is likely that visual processing is 'educated' by motor processing during visual practice, thus explaining superior intramodality learning.

The interpretation that enhanced motor competencies underlie APT is not the only one possible. Denying visual information during motor practice ensured that the motor-visual transfer cannot, even partially, be attributed to intramodality (visual) learning. However, as indicated in the Introduction, it is possible to explain the present APT effect as transfer from an enhanced ability to interpret the kinesthetic signals arising during motor practice to the visual task, i.e., as intermodal (kinesthetic-visual) and not motor-visual transfer. Although this interpretation does not invalidate the possible involvement of motor processes (see General discussion), a more clear-cut picture would emerge if we demonstrated that APT cannot be sufficiently explained as intermodal transfer. Our second experiment addressed this issue by manipulating the opportunity for active planning during motor practice.

\section{Experiment 2}

Since actions involve a planning or preparatory component as well as a kinesthetic feedback about the movement, both could be functional for the APT effect as observed in Experiment 1. To distinguish between these possibilities, we introduced a 'passive' kinesthetic learning condition in addition to the 'active' motor practice condition of Experiment 1. That is, one group planned and carried out arm movements, while a second group only received kinesthetic information about the movement. This was achieved by yoking a second passive lever to the apparatus. Thus, while leaving the experimental setup as similar as possible, the preparatory and efferent components of motor practice were separated from its kinesthetic (reafferent) component. Both groups received verbal feedback in the practice phase, but only the active group received the verbal command 
to produce a certain time ratio. Depending on the nature of APT, visual transfer performance should differ between the active and the passive condition.

\section{Method}

Participants. Thirty-two right-handed students (16 male, 16 female) at Ludwig-Maximilians-Universität, Munich, were paid for their participation. None of them had participated in Experiment 1.

Design. A between-subjects comparison was made between active and passive motor learning. Participants were tested in pairs of equal gender. One person in each pair was randomly assigned to active, the other to passive practice. A visual test followed the practice phase. A modification to the mapping between movements and percentage values was made since participants of Experiment 1 had indicated that they would be more comfortable with time ratios that correspond to increases or decreases in velocity of the second cycle. Thus, instructions and feedback were rephrased so that small ratios now reflected long second cycles and vice versa.

Motor task and apparatus. A second lever, on which a second participant could rest her/his arm, was yoked to the original lever, mounted $60 \mathrm{~cm}$ to its right. The two levers were connected at their tips with a rigid metal rod. The two ends of the metal rod had low-friction bearings, one of which was attached to the tip of the lever on the left, the other one to the tip of the lever on the right. Thus, whenever the person on the left (active participant, see Fig. 4) moved his or her lever by a given amount, the second lever would go through the exact same movement simultaneously. The right arm of the "passive" participant was attached to the lever in two places with Velcro tape. The active person had to produce the same two-cycle arm movements as used in Experiment 1, while the "passive" participant, who was also blindfolded, experienced only kinesthetic information about the movement that was carried out.

To verbally communicate the desired velocity ratios to the active participant while preventing the passive participant from listening, both participants wore head sets. Before each trial, the experimenter announced the desired percentage value at a low voice using a microphone. The amplified sound was routed to the headphone of the active participant, while the passive participant heard amplified white noise.

\section{Practice and test procedures}

Active practice. Active participants were asked to produce the same movement ratios used in Experiment 1, except that the active participant had to use more force to produce two-cycle movements within the required parameters, because the arm of the passive participant had to be moved via the yoked second lever. Instructions and feedback were given in terms of inverted duration ratios (interval $1 \times 100 /$ interval 2), since in Experiment 1 some participants had initially been confused by the task to translate a high percentage value into a movement with decreasing speed. For example, a movement with an $800 \mathrm{~ms}$ part (cycle 1) followed by a 1600 ms part (cycle 2) was now named as $50 \%$. This method, which effectively mapped percentage values to the velocity of cycle 2 relative to cycle 1 , proved to be less error prone. After being blindfolded, participants practiced several $100 \%$ movements before they carried out three blocks of 21 practice trials each, with on-line feedback about the actually produced timing.

Passive practice. Passive participants were instructed to relax their right arm, which rested on the second lever. They were also verbally introduced to the duration-ratio concept. Then, they were blindfolded and asked to guess what percentage value the active person had produced. Only on the first $100 \%$ trials did they know what the active person had intended to do. For all 63 practice trials, passive participants had to guess velocity ratios solely based on their kinesthetic impression of the movement. They knew that the range of prescribed ratios would roughly be between 50 and 200\%. Every six trials they were asked to communicate their guess to the experimenter. Feedback about the actually produced ratio was given verbally to both participants. Thus, active participants received information about the desired and executed timing, while passive participants received only kinesthetic feedback.

To ensure that the passive participant did not attempt to influence the movement carried out by the active person, the latter was asked to notify the experimenter whenever he or she felt that the passive partner pushed or pulled the lever. In such cases, the passive participants were reminded to relax their arm, and the trial

Fig. 4 Schematic drawing of the setup used in Experiment 2. During motor practice, the active (left $)$ and the passive (right) participant were blindfolded. Active participants performed timed two-cyclic movements on verbal command, while passive participants received only kinesthetic information during performance by virtue their lever being yoked to the active lever. The right arm of the passive participant was held by two Velcro strips around the lower arm (striped patches). After each trial, both participants were given verbal feedback about the actual timing produced. During the subsequent visual test, both participants judged the duration ratios of two-cycle sinusoidal bar movements that were displayed on an analog monitor. Note that in Experiment 1 only one lever was present and participants were tested individually

\section{Motor Practice}
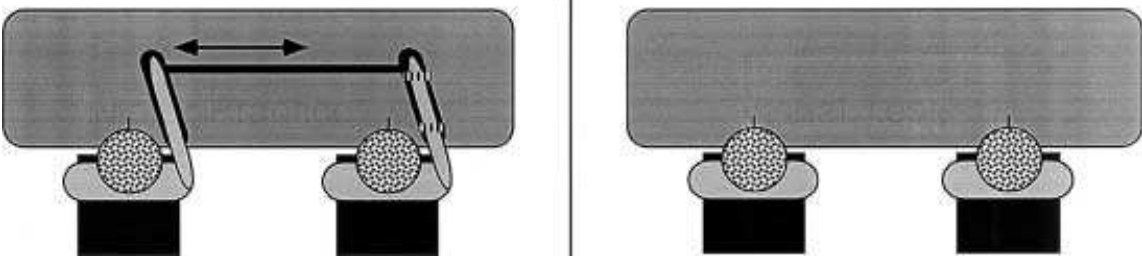
was repeated. This happened on average one to three times per pair during the entire practice phase.

Visual test. All participants received the same visual test. As in Experiment 1, they were shown the standard two-cycle movement with a $100 \%$ ratio first. Observers were asked to make ratio judgments for two blocks of 21 test trials each. The trials were identical to the ones used in Experiment 1 with regard to all stimulus parameters. The active and the passive participant watched the display in parallel (Fig. 4). On each trial, the complete two-cycle motion was presented twice before a $\%$ value had to be written on a prepared sheet of paper. Feedback was not provided.

Results

The visual test revealed no differences between the active and the passive group. The visual test data were analyzed according to the same procedure as in Experiment 1. The overall error $E$ was comparable in magnitude to the errors obtained in the first experiment. Active participants $(\mathrm{E}=14.33)$ and passive participants $(\mathrm{E}=14.87)$ did not differ significantly according to a repeated measures ANOVA $[F(1,45)=0.10, p=0.96]$. VE and CE measures showed the same pattern, that is, active and passive motor practice did not differ in their effect on constant or variable error during the visual test.

When compared to the control participants from Experiment 1, the active and the passive group produced smaller total errors (E), $F(1,45)=14.90, p<0.001$ for the active and $F(1,45)=15.24, p<0.001$ for the passive group. No differences between the first and the second block of the visual test were found.

\section{Discussion}

Active and passive participants did not differ in transfer performance, and both made smaller errors in the visual test than the control group of Experiment 1. Thus, kinesthetic feedback about the movement was sufficient to create the same amount of APT that was achieved when movements were planned, executed and felt. More precisely, the comparison between sensory information and the experimenter-provided feedback in the practice phase appears to be sufficient for APT. Thus, we have no grounds to assume that motor preparation or execution per se contribute to APT in the present task.

\section{General discussion}

We have, for the first time, demonstrated motor-perceptual effects on a longer time scale. Experiment 1 has demonstrated this APT for simple timed arm movements whose practice facilitates visual judgment of similar movement patterns. Experiment 1 also provided evidence for PAT or observational learning. The APT effect cannot be explained as an intramodal (visual-visual) transfer since motor practice was performed with eyes closed. In Experiment 2 we assessed the contribution of kinesthetic afferents during motor practice by comparing an active and a passive condition, where the "passive" participant could feel a movement performed by the "active" participant and both received verbal feedback about its time ratio. The passive group could not engage in motor planning since verbal commands were only given to the active participant. From the equivalent transfer of active and passive practice to the visual test in Experiment 2, we conclude that the relevant source of APT during motor practice is the availability of kinesthetic information together with feedback about its environmental effects. In contrast, motor preparatory or execution processes in the practice phase did not contribute to APT. Thus, explanations of APT as deriving solely from the efferent component of motor practice must be discarded, at least for the present task.

In the following, we relate APT back to the different theoretical frameworks that it supports. We focus on two main models, the notion of a structural overlap or common code between motor and visual representation (shared representations), and the notion of a matching process that is able to relate visual input in the judgment task to previously received kinesthetic signals (crossmodal processing). We favor this matching process as best suited to explain how practice in interpreting kinesthetic afferents may improve judgments of visual motion in the visual test (APT), and we entertain three conceivable explanations for it.

\section{Shared representations}

Within a representational framework, motor practice is thought to modify a memory structure that is sufficiently abstract to represent body movement as well as perceptual motion. This common code has the advantage of not requiring a translation between sensory inputs of different modalities, but also has the disadvantage of being abstract (see Prinz, 1992, 1997). While our results are predicted by the notion of common coding, they do not allow us to narrow down the code in much detail. While a specific code may exist, our experiment was not designed to examine its nature. One minimalistic interpretation of the common code in the present task would be to reduce it to timekeeping aspects. A general timekeeping mechanism could receive both kinesthetic and visual input. According to this interpretation, the transfer would have resulted from a refinement of the internal time representation that is available to all modalities. How plausible is this interpretation?

The literature on timing does indeed support the notion of amodal timekeepers (for a recent overview see Rosenbaum \& Collyer, 1998). In the first of a series of studies, Keele, Pokorny, Corcos, and Ivry (1985) found that the variances of repetitive tapping with finger or foot were correlated across subjects. They were also correlated with performance in a perceptual timing task, 
where participants judged whether the second of two intervals was shorter or longer than the standard first interval. This finding supports the idea of a common timing mechanism involved in a range of motor and perceptual tasks (see also Ivry \& Hazeltine, 1995; Rosenbaum, 1991, Ch. 8). Further, Ivry and Keele (1989) tested a range of neurological patients and found that only patients with cerebellar lesions were significantly impaired on both (motor and perceptual) tasks. The authors suggested that the underlying timing mechanism represents an isolable component, likely involving a loop between cortex and cerebellum, which can be employed by different motor and perceptual systems "when temporally predictive computations are needed" (Ivry \& Keele, p. 136). If such a timing representation exists, it most likely cannot not be accessed consciously by the observer. When asked after the experiment, none of the participants in our study reported the use of timekeeping strategies such as counting or metronome-like limb movements.

This framework would also explain possible effects of motor planning on visual judgment. However, given the absence of such effects in Experiment 2, we can focus on kinesthetic afferents here. Although differences between modalities in processing temporal information should not be dismissed (particularly between vision and audition, e.g., Kolers \& Brewster, 1985), the notion of a common timing mechanism that is accessible from kinesthetic and visual afferents is a plausible and parsimonious explanation of our APT effect. A key difference to the following cross-modal explanations is that, during the visual test, no reference to kinesthetic signals needs to be invoked. However, to evaluate this assumption is outside the scope of this article. Moreover, the main reason for us to seek for explanations beyond amodal timekeepers is that this explanation is specific for timing tasks and could thus not be applied to a wider range of tasks for which APT effects may be expected. Furthermore, additional assumptions would have to be made to accommodate the (well-documented) contribution of motor processes in motion perception.

\section{Cross-modal processing}

If we do not want to buy into the role of structural representations, APT can also be explained by processoriented frameworks. One such framework is the notion of transfer-appropriate processing (see Lee, 1988). It states that the compatibility between practice and transfer conditions determines the quality of the transfer. If we spell this idea out for the current experiment and relate it to cross-modal processing, this compatibility could reside in a 'visual-kinesthetic matching' or in a 'visuomotor-kinesthetic matching'. Both are considered in turn.

According to visual-kinesthetic matching, subjects match the novel visual stimuli to kinesthetic signals, which they have learned to interpret in terms of per- centages. Evidence for intermodal matching has been found already in 4-month-old infants. For example, Spelke (1976) simultaneously presented two films showing different rhythmic movement. A soundtrack was synchronized with one but not the other. Infants preferentially looked at the film that was synchronous with the sound, indicating an early capability to match visual and auditory modalities (for review see Bremner, 1994, Ch. 3). Further, visual-kinesthetic matching has been suggested to be involved in infants' imitation of facial expressions, where no intramodal (visual-visual) comparison is possible (Meltzoff \& Moore, 1997). In both of these cases of intermodal matching, however, sensory inputs from two modalities were present at some point, whereas in our visual test, participants would have had to generate expected kinesthetic signals from the visual input. Such visually induced kinesthetic images are certainly conceivable (Jeannerod, 1994, 1997). Our main discomfort with this explanation for APT is, however, that we would be left with a number of arguments for the involvement of visuomotor couplings in the visual task (see Introduction and Discussion of Experiment 1), but these couplings would not serve any function.

The second interpretation of transfer-appropriate processing, visuomotor-kinesthetic matching, is similar to the above but allows for the additional involvement of motor processes. In the visual test, visual-kinesthetic matching may be mediated via motor encoding. That is, the required visual judgment would be accomplished by the intertwined operation of two processes. First, the visual pattern activates not only visual but also motor representations (visuomotor couplings). Second, access to the learned mapping of environmental outcomes (time ratios) is gained via expected kinesthetic afferents that are part of the motor representation. Whereas the mechanism for visual-kinesthetic induction in our earlier explanation may appear somewhat vague, motor-kinesthetic induction is a variant of the general concept of efference copy or corollary discharge (see Jeannerod, 1997, Ch. 6). Premotor areas have a rich output to the parietal lobe, which provides a likely substrate of both motor-kinesthetic priming (as suggested here) and motor-visual priming (see Craighero et al., 1999). Thus, visuomotor couplings may further involve expected kinesthetic afferents, which our participants have already learned to interpret during motor (active or passive) practice. This explanation does not preclude more direct links between vision and proprioception or even between vision and environmental outcome, rather, such links may be supplemented or even formed by concurrent motor involvement.

In addition to visuomotor-kinesthetic matching, it is conceivable that 'motor encoding' may have been involved in our 'passive' practice condition. Although we have discarded this possibility before, a closer examination reveals that participants may well have engaged in preparing a motor representation that was not fully specified (two cycles with unspecified or default duration 
of the second cycle). They may have 'filled-in' this missing parameter as soon as it became available kinesthetically. Evidence for such partial motor preparation has been reported by Favilla, Hening and Ghez (1989; see also Favilla \& De Cecco, 1996). Furthermore, premotor neurons do not only respond to visual motion input (mirror neurons), but also to somatosensory stimuli (G. Rizzolatti, personal communication). Thus, a physiological basis for the proposed filling-in does indeed exist. This possibility may be described as kinesthetically guided motor imagery, which could have contributed to APT by forming links between motor processes and environmental outcome in the practice phase. These motor links may subsequently augment or even bypass the visuomotor-kinesthetic-outcome links in the visual test.

To summarize, we have offered three possible explanations for our APT effect. The notion of a timekeeping mechanism that can be addressed by kinesthetic and visual input appears to be the most parsimonious account for the present results; however, this account is limited to timing tasks. Although our visual display provided temporal information, it also provided displacement and velocity information that could have been used to inform participants' judgments. The alternative explanations (visual-kinesthetic and visuomotor-kinesthetic matching) can be applied to a wider spectrum of tasks, including velocity-based judgments, and thus continue to be conceivable candidates in future studies of APT.

The evidence for APT has a number of consequences for our understanding of motor learning. The acquisition of motor skill cannot be modeled as an open-loop process in which visual perception informs the setting of motor parameters. Instead, APT suggests that it be modeled as a closed loop in which motor learning educates perception as much as vice versa. For training situations this might suggest that the novice should not start out by observing the expert, but rather by attempting to execute the action before observing. The benefit of observation should be increased. In this context, our findings encourage the study of training programs that put a strong emphasis on motor execution also when the goal is to become a perceptual expert, such as a referee.

To further investigate the role of kinesthetic feedback during motor practice, one could reduce motor practice to its preparatory component using motor imagery instructions (Jeannerod, 1994, 1997). This option was not available for the current task, where motor practice involved experimenter-supplied feedback on performance. Wohlschläger (2000) has demonstrated effects of motor preparation per se on concurrent visual judgment, and it would be interesting to explore whether such effects can also be found on the longer time scale of APT paradigms. As we have seen, however, a full explanation of APT effects requires consideration of intermodal interaction processes during practice as well as during transfer. That is, even a well-located source of APT effects in the practice phase may leave us with more than one viable explanation for transfer performance.
The importance of further investigating APT is also evident in the light of recent findings suggesting that intended actions shape our perception of complex motion. A ball that is propelled by a human actor looks very natural when it continues to accelerate after it has already left the thrower's hand (Hecht and Bertamini, 2000). Here the past experience of throwing actions exerts an influence on experienced ball movement, even defying the laws of classical mechanics. Thus, the further study of APT may hold the key to understanding how the visual system represents events.

Acknowledgements We wish to thank Heiner Deubel and Henryk Milewski for programming support, Fiorello Banchi for fabricating the two-lever apparatus, and Nicola Korherr for her support in recruiting participants and running the experiments.

\section{References}

Arbib, M. A. (1997). Modeling visuomotor transformations. In F. Boller \& J. Grafman (Series Eds.) \& M. Jeannerod (Vol. Ed.), Handbook of Neuropsychology, Vol. 11, Section 16: Action and cognition (pp. 65-90). Amsterdam: Elsevier.

Bandura, A. (1986). Social foundations of thought and action: a social cognitive theory. Englewood Cliffs, NJ: Prentice-Hall.

Bekkering, H. (in press). Imitation: common mechanisms in the observation and execution of finger and mouth movements. In A. N. Meltzoff \& W. Prinz (Eds.), The imitative mind: development, evolution and brain bases. Cambridge, MA: Cambridge University Press.

Brass, M., Bekkering, H., \& Prinz, W. (in press). Movement observation affects movement execution in a simple response task. Acta Psychologica.

Bremner, J. G. (1994). Infancy (2nd ed.). Oxford, UK: Blackwell.

Byrne, R. W., \& Russon, A. E. (1998). Learning by imitation: a hierarchical approach. Behavioral and Brain Sciences, 21, 667721.

Carroll, W. A., \& Bandura, A. (1990). Representational guidance of action production in observational learning: a causal analysis. Journal of Motor Behavior, 22, 85-97.

Craighero, L., Fadiga, L., Rizzolatti, G., \& Umiltà, C. (1999). Action for perception: a motor-visual attentional effect. Journal of Experimental Psychology: Human Perception and Performance, 25, 1673-1692.

Craighero, L., Fadiga, L., Umiltà, C. A., \& Rizzolatti, G. (1996). Evidence for a visuomotor priming effect. NeuroReport, $8,347-$ 349.

DiPellegrino, G., Fadiga, L., Fogassi, L., Gallese, V., \& Rizzolatti, G. (1992). Understanding motor events: a neurophysiological study. Experimental Brain Research, 91, 176-180.

Favilla, M., Hening, W., \& Ghez, C. (1989). Trajectory control in targeted force impulses. VI. Independent specification of response amplitude and direction. Experimental Brain Research, 75, 280-294.

Favilla, M., \& De Cecco, E. (1996). Parallel direction and extent specification of planar reaching arm movements in humans. Neuropsychologia, 34, 609-613.

Gallese, V., \& Goldman, A. (1998). Mirror neurons and the simulation theory of mind-reading. Trends in Cognitive Sciences, 2, 493-501.

Grezes, J., Costes, N., \& Decety, J. (1998). Top-down effect of strategy on the perception of human biological motion: a PET investigation. Cognitive Neuropsychology, 15, 553-582.

Hecht, H., \& Bertamini, M. (2000). Understanding projectile acceleration. Journal of Experimental Psychology: Human Perception and Performance, 26, 730-746.

Hommel, B., \& Prinz, W. (1997, Eds.). Theoretical issues in stimulus-response compatibility. Amsterdam: Elsevier. 
Ishimura, G., \& Shimojo, S. (1994, May). Voluntary action captures visual motion. Poster presented at the annual meeting of the Association for Research in Vision and Ophthalmology, Sarasota, FL.

Ivry, R. B., \& Hazeltine, R. E. (1995). Perception and production of temporal intervals across a range of durations: evidence for a common timing mechanism. Journal of Experimental Psychology: Human Perception and Performance, 21, 3-18

Ivry, R. B., \& Keele, S. W. (1989). Timing functions of the cerebellum. Journal of Cognitive Neuroscience, 1, 136-152

Jeannerod, M. (1994). The representing brain: neural correlates of motor intention and imagery. Behavioral and Brain Sciences, 17, 187-245.

Jeannerod, M. (1997). The cognitive neuroscience of action. Oxford: Blackwell.

Jeannerod, M., Arbib, M. A., Rizzolatti, G., \& Sakata, H. (1995). Grasping objects: the cortical mechanisms of visuomotor transformation. Trends in Neurosciences, 18, 314-320.

Jeannerod, M., \& Decety, J. (1995). Mental motor imagery: a window into the representational stages of action. Current Opinion in Neurobiology, 5, 727-732.

Keele, S. W., Pokorny, R. A., Corcos, D. M., \& Ivry, R. (1985). Do perception and motor production share common timing mechanisms: a correlational analysis. Acta Psychologica, 60, 173-191.

Kolers, P. A., \& Brewster, J. M. (1985). Rhythms and responses. Journal of Experimental Psychology: Human Perception and Performance, 11, 150-167.

Lee, T. D. (1988). Transfer-appropriate processing: a framework for conceptualizing practice effects in motor learning. In O. G. Meijer \& K. Roth (Eds.), Complex motor behaviour: 'The' motor-action controversy (pp. 201-215). Amsterdam: Elsevier.

McCullagh, P., Weiss, M. R., \& Ross, D. (1989). Modeling considerations in motor skill acquisition and performance: an integrated approach. Exercise and Sport Sciences Reviews, $17,475-513$.

Meltzoff, A. N., \& Moore, M. K. (1997). Explaining facial imitation: a theoretical model. Early Development and Parenting, 6, 179-192.

Meltzoff, A. N., \& Prinz, W. (Eds.) (in press). The imitative mind: development, evolution and brain bases. Cambridge, MA: Cambridge University Press.

Müsseler, J., \& Hommel, B. (1997). Blindness to response-compatible stimuli. Journal of Experimental Psychology: Human Perception and Performance, 23, 861-872.

Müsseler, J. (1999). How independent from action control is perception? An event-coding account for more equally-ranked crosstalks. In G. Aschersleben, T. Bachmann \& J. Müsseler
(Eds.), Cognitive contributions to the perception of spatial and temporal events (pp. 121-147). Amsterdam: Elsevier.

Prinz, W. (1992). Why don't we perceive our brain states? European Journal of Cognitive Psychology, 4, 1-20.

Prinz, W. (1997). Perception and action planning. European Journal of Cognitive Psychology, 9, 129-154.

Prinz, W. (in press). Experimental approaches to imitation. In A. N. Meltzoff \& W. Prinz (Eds.), The imitative mind: development, evolution and brain bases. Cambridge, MA: Cambridge University Press.

Rizzolatti, G., Fadiga, L., Gallese, V., \& Fogassi, L. (1996). Premotor cortex and the recognition of motor actions. Cognitive Brain Research, 3, 131-141.

Rosenbaum, D. A. (1991). Human motor control. New York: Academic Press.

Rosenbaum, D. A., \& Collyer, C. E. (1998, Eds.). Timing of behavior. Cambridge, MA: MIT Press.

Schmidt, R. A. (1988). Motor control and learning: a behavioral emphasis. Champaign, IL: Human Kinetics.

Shiffrar, M., \& Freyd, J. J. (1993). Apparent motion of the human body. Psychological Science, 1, 257-264.

Spelke, E. S. (1976). Infant's intermodal perception of events. Cognitve Psychology, 8, 553-560.

Tucker, M., \& Ellis, R. (1998). On the relations between seen objects and components of potential actions. Journal of Experimental Psychology: Human Perception and Performance, 24, 830-846.

Viviani, P. \& Stucchi, N. (1992a). Motor-perceptual interactions. In G. E. Stelmach \& J. Requin (Eds.), Tutorials in motor behavior: II (pp. 229-248). Amsterdam: North-Holland.

Viviani, P., \& Stucchi, N. (1992b). Biological movements look uniform: evidence for motor-perceptual interactions. Journal of Experimental Psychology: Human Perception and Performance, $18,602-623$.

Vogt, S. (1995). On relations between perceiving, imagining and performing in the learning of cyclical movement sequences. British Journal of Psychology, 86, 191-216.

Vogt, S. (1996). Imagery and perception-action mediation in imitative actions. Cognitive Brain Research, 3, 79-86.

Vogt, S. (in press-a). Visuomotor couplings in object-oriented and imitative actions. In A. N. Meltzoff \& W. Prinz (Eds.), The imitative mind: development, evolution and brain bases. Cambridge, MA: Cambridge University Press.

Vogt, S. (in press-b). Dimensions of imitative perception-action mediation. In K. Dautenhahn \& C. Nehaniv (Eds.), Imitation in animals and artifacts. Boston, MA: MIT Press.

Wohlschläger, A. (2000). Visual motion priming by invisible actions. Vision Research, 40, 925-930. 